\title{
Comparison of Lipid Profile in Patients With and Without Subclinical Hypothyroidism
}

Mishal Ejaz ${ }^{1}$, Pardeep Kumar ${ }^{2}$, Murlidhar Thakur ${ }^{3}$, Parkash Bachani ${ }^{4}$, Sidra Naz ${ }^{5}$, Kirshan Lal $^{6}$, Wajeeha Shahid $^{2}$, Simra Shahid ${ }^{2}$, Maha Jahangir ${ }^{7}$, Amber Rizwan ${ }^{8}$

1. Internal Medicine, Ziauddin University, Karachi, PAK 2. Internal Medicine, Jinnah Sindh Medical University, Karachi, PAK 3. Internal Medicine, Chandka Medical College, Larkana, PAK 4. Internal Medicine, Liaquat University of Medical and Health Sciences, Jamshoro, PAK 5. Internal Medicine, University of Health Sciences, Lahore, PAK 6. Internal Medicine, Ghulam Muhammad Mahar Medical College, Sukkur, PAK 7. Internal Medicine, Dow University of Health Sciences, Karachi, PAK 8. Family Medicine, Jinnah Post Graduate Medical Center, Karachi, PAK

Corresponding author: Maha Jahangir, jahangirmaha@yahoo.com

\section{Abstract}

Introduction: Thyroid hormone affects lipid metabolism. Various studies have shown a contradictory relationship between lipid profile (LP) and subclinical hypothyroidism (SCH). Currently, there is a scarcity of regional data on the relationship between $\mathrm{LP}$ and $\mathrm{SCH}$.

Methods: This longitudinal study was conducted in the internal medicine and cardiology units of a tertiary care hospital in Pakistan from September 2019 to March 2021. A total of 900 participants, of either gender and between the ages of 40 to 70 years, were enrolled in the study. Blood samples were sent to the laboratory to determine lipid and thyroid parameters. Participants were divided into two groups based on the presence of SCH.

Results: In our study, 179 (19.8\%) participants had SCH. Total cholesterol (TC) and low-density lipoprotein (LDL) was significantly higher in participants with SCH compared to participants without SCH $(228.41 \pm$ $35.21 \mathrm{mg} / \mathrm{dL}$ vs. $171.21 \pm 30.21 \mathrm{mg} / \mathrm{dL}$; p-value: $<0.00001)$ and $(131.65 \pm 28.22 \mathrm{mg} / \mathrm{dL}$ vs. $89.26 \pm 18.52$ $\mathrm{mg} / \mathrm{dL}$; p-value: $<0.0001$ ), respectively.

Conclusion: In conclusion, this study found an increased incidence of dyslipidemias in patients with SCH. It is associated with elevated TC and LDL levels, which are risk factors for cardiovascular disease and mortality.

Review began $07 / 22 / 2021$ Review ended 08/12/2021 Published 08/19/2021

\section{(c) Copyright 2021}

Ejaz et al. This is an open access article distributed under the terms of the Creative Commons Attribution License CC-BY 4.0., which permits unrestricted use, distribution, and reproduction in any medium, provided the original author and source are credited.
Categories: Cardiology, Endocrinology/Diabetes/Metabolism, Internal Medicine

Keywords: lipid profile, hypothryroidism, subclinical hypothryroidism, sch, thyroxine

\section{Introduction}

In subclinical hypothyroidism (SCH), thyroid-stimulating hormone (TSH) levels are elevated in the blood, but serum free thyroxine (FT4) levels are normal. It is rather prevalent, affecting about $10 \%$ of women over the age of 55 [1]. SCH is linked to a higher risk of heart failure, coronary artery disease events, and coronary heart disease-related mortality. In addition, patients with $\mathrm{SCH}$ in their middle years may experience cognitive impairment, nonspecific symptoms such as weariness, and mood swings [2]. Other cardiovascular risk factors, such as blood pressure changes and increased atherosclerosis have been related to $\mathrm{SCH}$. A change in lipid profile (LP) is another consequence of SCH [3]. Thyroid hormone affects lipid metabolism, and numerous studies have found that lipid levels increase as TSH levels rise [3]. Serum low-density lipoprotein-C (LDL-C) levels were observed to be higher in SCH patients in several investigations. Some of them discovered greater serum total cholesterol (TC) levels in SCH patients, whereas others discovered lower serum TC levels in SCH patients [4]. Changes in blood high-density lipoprotein-C (HDL-C) and triglyceride (TG) levels under SCH have also yielded contradictory results $[5,6]$.

Currently, there is a scarcity of regional data on the relationship between lipid levels and SCH. To analyze and identify patients at risk of cardiovascular events, it is crucial to understand the link between lipid readings and $\mathrm{SCH}$.

\section{Materials And Methods}

This longitudinal study was conducted in the tertiary care hospital's internal medicine and cardiology unit from September 2019 to March 2021. Community-dwelling participants $(n=900)$ of either gender, between the ages of 40 to 70 years, who were visiting the outpatient department for routine checkups, were enrolled in the study. The entire procedure was explained to the participants and their consent was taken. Participants were enrolled via consecutive convenient non-probability sampling. Ethical review board approval was taken from the institute before the start of the study. Participants with known thyroid dysfunction or participants on statin medication were excluded from the study. Furthermore, subjects on 


\section{Cureus}

levothyroxine or antithyroid drugs, amiodarone or lithium, were also excluded from the study in addition to patients with terminal illness and patients who underwent recent surgery (within the last four weeks).

After enrollment, detailed history of the participant was taken and noted in a self-structured questionnaire. After the history, consent was taken again to withdraw 5-mL blood from the cubital vein via phlebotomy in two different vials. Blood was sent to the laboratory to determine lipid and thyroid parameters. Lipid parameters included TC, LDL-C, HDL-C, and TG levels. Thyroid parameters included TSH, FT4, and free triiodothyronine (FT3). SCH was defined as TSH levels between 5 and $10 \mathrm{mIU} / \mathrm{L}$ with normal thyroid hormones [7]. Participants were divided into two groups based on the presence or absence of SCH.

Data analysis was done using the Statistical Package for Social Sciences ${ }^{\circledR}$ software, version 23.0 (SPSS; IBM Corp., Armonk, NY, USA). Continuous variables such as age, TSH. T3, T4, low-density lipoprotein (LDL), high-density lipoprotein (HDL), TC, and TG were presented as mean and standard deviation. Categorical variables such as gender and the presence of SCH were presented by percentages and frequencies. Mean lipid values for groups with and without SCH were compared using an independent t-test. A p-value of less than 0.05 was considered statistically significant.

\section{Results}

In our study, 179 (19.8\%) participants had SCH. SCH was more common in females and participants with higher body mass index (BMI). TSH was significantly higher in patients with SCH compared to participants without SCH (6.58 $\pm 1.15 \mathrm{mIU} / \mathrm{L}$ vs. $3.12 \pm 0.56 \mathrm{mIU} / \mathrm{L}$; p-value: 0.0001$)$ (Table 1).

\begin{tabular}{|c|c|c|c|}
\hline Characteristics & Participants with SCH (n = 179) & Participants without SCH $(n=721)$ & p-value \\
\hline Age in years (Mean $\pm S D$ ) & $53.2 \pm 14.6$ & $52.7 \pm 13.9$ & NS \\
\hline Female & $112(62.5 \%)$ & $342(47.4 \%)$ & 0.0002 \\
\hline Hypertension & $152(84.9 \%)$ & $593(83.3 \%)$ & NS \\
\hline Smoking & $56(31.2 \%)$ & 237 (32.8\%) & NS \\
\hline Type 2 DM & $81(45.2 \%)$ & $346(47.9 \%)$ & NS \\
\hline BMI greater than $25 \mathrm{~kg} / \mathrm{m}^{2}$ & $62(34.6 \%)$ & $166(23.0 \%)$ & 0.001 \\
\hline TSH (mlU/L) & $6.58 \pm 1.15$ & $3.12 \pm 0.56$ & $<0.0001$ \\
\hline FT3 (pg/dL) & $342.61 \pm 91.65$ & $335.43 \pm 89.21$ & NS \\
\hline FT4 (ng/dL) & $1.41 \pm 0.33$ & $1.46 \pm 0.37$ & NS \\
\hline
\end{tabular}

\section{TABLE 1: Characteristics of the participants}

BMI: body mass index; DM: diabetes mellitus; FT3: free triiodothyronine; FT4: free thyroxine; kg/m2: kilogram per square meter; mIU/L: milliinternational units per liter; ng/dL: nanograms per decilitre; NS: nonsignificant; pg/dL: picogram/decilitre; SCH: subclinical hypothyroidism; SD: standard deviation; TSH: thyroid-stimulating hormone.

TC and LDL were significantly higher in participants with SCH compared to participants without SCH $(228.41 \pm 35.21 \mathrm{mg} / \mathrm{dL}$ vs. $171.21 \pm 30.21 \mathrm{mg} / \mathrm{dL} ; \mathrm{p}$-value: $<0.00001)$ and $(131.65 \pm 28.22 \mathrm{mg} / \mathrm{dL}$ vs. $89.26 \pm$ $18.52 \mathrm{mg} / \mathrm{dL}$; p-value: $<0.0001$ ), respectively (Table 2). 


\section{Cureus}

\begin{tabular}{|c|c|c|c|}
\hline Lipid parameters (mg/dL) & Participants with SCH $(n=179)$ & Participants without SCH $(n=721)$ & $p$-value \\
\hline TC & $228.41 \pm 35.21$ & $171.21 \pm 30.21$ & $<0.0001$ \\
\hline HDL & $36.42 \pm 10.12$ & $37.12 \pm 9.72$ & 0.39 \\
\hline LDL & $131.65 \pm 28.22$ & $89.26 \pm 18.52$ & $<0.0001$ \\
\hline TG & $122.61 \pm 31.22$ & $120.19 \pm 31.01$ & 0.35 \\
\hline
\end{tabular}

\section{TABLE 2: Comparison of lipid parameters in participants with and without SCH}

LDL: low-density lipoprotein; HDL: high-density lipoprotein; mg/dL: milligram/deciliter; SCH: subclinical hypothyroidism; TC: total cholesterol; TG: triglyceride.

\section{Discussion}

This study compared lipid profiles among individuals with SCH and healthy controls. According to our results, individuals with SCH were more likely to be females and more likely to have a BMI of more than 25 $\mathrm{kg} / \mathrm{m} 2$. It was associated with hypercholesteremia and higher LDL levels as compared to the healthy controls. There was no significant difference seen in HDL and TG levels.

Vierhapper et al. conducted a study on patients with euthyroidism, $\mathrm{SCH}$, and overt hypothyroidism and observed similar LDL-C levels in euthyroid and SCH patients, and increased levels in overt hypothyroidism only [8]. Hueston et al. also stated that SCH is not associated with abnormalities in serum cholesterol or TG levels [9]. However, a study conducted in India showed similar results that the increase in LDL, TG, and TC is dependent on the increase in TSH levels. Hence, hyperlipidemia is more common in patients with SCH [10]. A cross-sectional study on 25,862 individuals in Colorado reported that changes in TSH levels were directly related to changes in lipid levels [11]. A meta-analysis done on data from 1990 to 2014 found that serum cholesterol, LDL-C, and TG were found to be higher in patients with SCH and quoted that the discrepancies in previous studies might have been a result of confounding factors [4].

The association between cholesterol levels and $\mathrm{SCH}$ is of great significance as dyslipidemias are associated with increased risk of hypertension, cardiovascular disease, and stroke incidence [3,12]. SCH is even considered a strong indicator of atherosclerosis and risk of myocardial infarction in elderly women [6]. Hence, lipid levels should be routinely monitored in patients with SCH to timely identify any derangements to prevent any associated adverse outcomes.

To correct the underlying cause of dyslipidemias in $\mathrm{SCH}$, treatment with thyroxine may be considered; however, the beneficence of its use on LP is still a matter of debate. Several investigations carried out to observe the outcome of thyroxine supplementation in patients with SCH have reported a reduction in the levels of TC and LDL levels [13-16]. Meier et al. reported approximately 9-31\% risk reduction of cardiovascular mortality in patients receiving thyroxine supplementation [13]. However, some authors have reported no significant reduction in TC and LDL levels after treatment with thyroxine [17-19]. Two of the factors that could be influencing the variance in the response of thyroxine therapy could be the baseline levels of TC and TSH levels before the start of the treatment. Patients with TSH levels $>10 \mathrm{uU} / \mathrm{ml}$ or TC $>240$ $\mathrm{mg} / \mathrm{dl}$ have shown a significant reduction in the levels of TC and LDL-C with thyroxine as compared to others [20]. However, inconsistencies in these results warrant further investigation on the use of thyroxine in patients with $\mathrm{SCH}$ for dyslipidemias.

To the best of our knowledge, this is the first study from this region to study the relation between lipid parameters and SCH. However, since the study was conducted in a single institute, care should be taken while inferring the result to the general population. Secondly, since it was a case-control study, a definite association could not be established. A large-scale prospective study is needed to confirm the association between dyslipidemia and $\mathrm{SCH}$.

\section{Conclusions}

In conclusion, this study found an increased incidence of dyslipidemias in patients with SCH. SCH is associated with elevated TC and LDL levels, which are risk factors for cardiovascular disease and mortality. To reduce the adverse outcomes associated with these findings, adequate monitoring of lipid profile is required along with treatment of the underlying cause in such patients. Thyroxine is one of the proposed interventions, but the evidence of its benefit is controversial.

\section{Additional Information}




\section{Disclosures}

Human subjects: Consent was obtained or waived by all participants in this study. Jinnah Sindh Medical University issued approval JSMU/IRB/2019/17. Animal subjects: All authors have confirmed that this study did not involve animal subjects or tissue. Conflicts of interest: In compliance with the ICMJE uniform disclosure form, all authors declare the following: Payment/services info: All authors have declared that no financial support was received from any organization for the submitted work. Financial relationships: All authors have declared that they have no financial relationships at present or within the previous three years with any organizations that might have an interest in the submitted work. Other relationships: All authors have declared that there are no other relationships or activities that could appear to have influenced the submitted work.

\section{References}

1. Redford C, Vaidya B: Subclinical hypothyroidism: should we treat? . Post Reprod Health. 2017, 23:55-62. $10.1177 / 2053369117705058$

2. Biondi B, Cappola AR, Cooper DS: Subclinical hypothyroidism: a review. JAMA. 2019, 322:153-60. 10.1001/jama.2019.9052

3. Delitala AP, Fanciulli G, Maioli M, Delitala G: Subclinical hypothyroidism, lipid metabolism and cardiovascular disease. Eur J Intern Med. 2017, 38:17-24. 10.1016/j.ejim.2016.12.015

4. Liu XL, He S, Zhang SF, et al.: Alteration of lipid profile in subclinical hypothyroidism: a meta-analysis . Med Sci Monit. 2014, 20:1432-41. 10.12659/MSM.891163

5. Iqbal A, Jorde R, Figenschau Y: Serum lipid levels in relation to serum thyroid-stimulating hormone and the effect of thyroxine treatment on serum lipid levels in subjects with subclinical hypothyroidism: the Tromsø study. J Intern Med. 2006, 260:53-61. 10.1111/j.1365-2796.2006.01652.x

6. Hak AE, Pols HA, Visser TJ, Drexhage HA, Hofman A, Witteman JC: Subclinical hypothyroidism is an independent risk factor for atherosclerosis and myocardial infarction in elderly women: the Rotterdam study. Ann Intern Med. 2000, 132:270-8. 10.7326/0003-4819-132-4-200002150-00004

7. Voigtländer R, Führer D: Subclinical hypothyroidism - laboratory finding or disease? (Article in German) . Dtsch Med Wochenschr. 2016, 141:1134-6. 10.1055/s-0042-107439

8. Vierhapper H, Nardi A, Grösser P, Raber W, Gessl A: Low-density lipoprotein cholesterol in subclinical hypothyroidism. Thyroid. 2000, 10:981-4. 10.1089/thy.2000.10.981

9. Hueston WJ, Pearson WS: Subclinical hypothyroidism and the risk of hypercholesterolemia . Ann Fam Med. 2004, 2:351-5. 10.1370/afm.79

10. Asranna A, Taneja RS, Kulshreshta B: Dyslipidemia in subclinical hypothyroidism and the effect of thyroxine on lipid profile. Indian J Endocrinol Metab. 2012, 16:S347-9. 10.4103/2230-8210.104086

11. Canaris GJ, Manowitz NR, Mayor G, Ridgway EC: The Colorado thyroid disease prevalence study . Arch Intern Med. 2000, 160:526-34. 10.1001/archinte.160.4.526

12. Alloubani A, Nimer R, Samara R: Relationship between hyperlipidemia, cardiovascular disease and stroke: a systematic review. [PREPRINT]. Curr Cardiol Rev. 2020, 10.2174/1573403X16999201210200342

13. Meier C, Staub JJ, Roth CB, et al.: TSH-controlled L-thyroxine therapy reduces cholesterol levels and clinical symptoms in subclinical hypothyroidism: a double blind, placebo-controlled trial (Basel Thyroid Study). J Clin Endocrinol Metab. 2001, 86:4860-6. 10.1210/jcem.86.10.7973

14. Caraccio N, Ferrannini E, Monzani F: Lipoprotein profile in subclinical hypothyroidism: response to levothyroxine replacement, a randomized placebo-controlled study. J Clin Endocrinol Metab. 2002, 87:15338. 10.1210/jcem.87.4.8378

15. Yildirimkaya M, Ozata M, Yilmaz K, Kilinç C, Gündoğan MA, Kutluay T: Lipoprotein(a) concentration in subclinical hypothyroidism before and after levo-thyroxine therapy. Endocr J. 1996, 43:731-6. 10.1507/endocrj.43.731

16. Arem R, Escalante DA, Arem N, Morrisett JD, Patsch W: Effect of L-thyroxine therapy on lipoprotein fractions in overt and subclinical hypothyroidism, with special reference to lipoprotein(a). Metabolism. 1995, 44:1559-63. 10.1016/0026-0495(95)90075-6

17. Nilsson G, Nordlander S, Levin K: Studies on subclinical hypothyroidism with special reference to the serum lipid pattern. Acta Med Scand. 1976, 200:63-7. 10.1111/j.0954-6820.1976.tb08197.x

18. Cooper DS, Halpern R, Wood LC, Levin AA, Ridgway EC: L-thyroxine therapy in subclinical hypothyroidism. A double-blind, placebo-controlled trial. Ann Intern Med. 1984, 101:18-24. 10.7326/0003-4819-101-1-18

19. Bell GM, Todd WT, Forfar JC, et al.: End-organ responses to thyroxine therapy in subclinical hypothyroidism. Clin Endocrinol (Oxf). 1985, 22:83-9. 10.1111/j.1365-2265.1985.tb01068.x

20. Efstathiadou Z, Bitsis S, Milionis HJ, Kukuvitis A, Bairaktari ET, Elisaf MS, Tsatsoulis A: Lipid profile in subclinical hypothyroidism: is L-thyroxine substitution beneficial?. Eur J Endocrinol. 2001, 145:705-10. 10.1530/eje.0.1450705 